Title

\title{
MODY patients exhibit shorter telomere length than non-diabetic subjects
}

\section{Authors}

Andrea L. Millan ${ }^{1,2} \mid$ Sofía I. Trobo² $\mid$ Alejandro de Dios ${ }^{3} \mid$ Martina Cerrato García $^{2}$ | María S. Pérez $^{5}$ | Gloria E. Cerrone ${ }^{1,2}$ | Gustavo D. Frechtel ${ }^{1,2,3}$ | Ariel P. López ${ }^{1,2,4}$

\section{Affiliations}

${ }^{1}$ Instituto de Inmunología, Genética y Metabolismo (INIGEM-UBA-CONICET). Facultad de Farmacia y Bioquímica. Universidad de Buenos Aires, Laboratorio de Diabetes y Metabolismo, Buenos Aires, AR.

${ }^{2}$ Facultad de Farmacia y Bioquímica, Universidad de Buenos Aires, Cátedra de Genética y Biología Molecular, Buenos Aires, AR.

${ }^{3}$ Hospital de Clínicas José de San Martín. Facultad de Medicina, Universidad de Buenos Aires, División Nutrición, Buenos Aires, AR.

${ }^{4}$ Hospital de Clínicas José de San Martín. Facultad de Medicina, Universidad de Buenos Aires, Programa de Biología Molecular, Buenos Aires, AR.

${ }^{5}$ Laboratorio Manlab, Buenos Aires, AR.

\section{Correspondence}

Andrea L. Millan. Instituto de Inmunología Genética y Metabolismo (INIGEM-UBACONICET). Av. Córdoba 2351, Buenos Aires, AR

Email: lic.andreamillan@gmail.com

This article has been accepted for publication and undergone full peer review but has not been through the copyediting, typesetting, pagination and proofreading process which may lead to differences between this version and the Version of Record. Please cite this article as doi: 10.1002/dmrr.3374 


\begin{abstract}
Aims: Given the increasing evidence supporting the association between telomere shortening and diabetes, the aim of the present work was to establish whether MODY patients suffer a reduction in TL due to oxidative stress produced by chronic hyperglycemia, despite not presenting insulin resistance or inflammation.

Materials and Methods: We analyzed clinical and biochemical parameters in 35 MODY2 and 12 MODY3 patients compared with 48 Control subjects. The absolute telomere length (aTL) of peripheral blood leukocytes was measured using the quantitative polymerase chain reaction (qPCR).

Results: A significant negative correlation was observed between aTL and age in the whole population, among MODY patients and in each subtype studied, MODY2 and MODY3, which allowed us to validate the method. We found, for the first time, that MODY patients have shorter aTL with respect to non-diabetic Controls (6.49 $\pm 3.31 \mathrm{Kbp}$ vs $11.13 \pm 7.82 \mathrm{Kbp}$, $\mathrm{p}=0.006)$. However, no differences were found between MODY2 and MODY3. Additionally, aTL showed a negative correlation with duration of the disease and FPG levels in MODY patients in general and also with HbA1c in MODY2 patients in particular.

Conclusions: It can be thus inferred that both MODY types present telomere shortening, which, at least partly, responds to HbA1c and FPG levels. These findings suggest comparable mechanisms underlying the attrition of TL. Taken together, our results on aTL in MODY patients may provide a parameter relatively easy and inexpensive to quantify in order to measure the impact of high glucose levels and potentially carry out antidiabetic treatment with stricter targets.
\end{abstract}

\title{
KEYWORDS
}

Hyperglycemia, MODY, Oxidative Stress, Telomere length 


\section{1 | INTRODUCTION}

Maturity-onset diabetes of the young (MODY) is a monogenic disorder characterized by autosomal dominant inheritance which usually develops in childhood, adolescence or young adulthood. ${ }^{1,2}$ It has been established that MODY represents 1-5\% of all cases of diabetes and is often misdiagnosed as type 1 diabetes (T1D) or type 2 diabetes (T2D). ${ }^{1,3,4}$

MODY is caused by mutations in different genes resulting in pancreatic $\beta$-cell dysfunction. ${ }^{5,6}$ There are 14 subtypes of MODY depending of the genes involved, MODY2 and MODY3 being the most frequent genotypes. MODY2 is caused by mutations in the glucokinase (GCK) gene, while MODY3 is caused by mutations in the hepatic nuclear factor (HNF1A) gene. ${ }^{2}$ The clinical characteristics of the different subtypes oscillate between a mild hyperglycemic state with good clinical prognosis (MODY2) to a more severe hyperglycemic state accompanied by early chronic complications (MODY3). Both subtypes present alterations in insulin secretion as a primary defect, without insulin resistance. ${ }^{7-9}$

Telomeres are nucleic-protein complexes located at the end of eukaryotic chromosomes, composed of several tandem repeats of a non-coding DNA hexanucleotide (TTAGGG in mammals) and associated proteins. Telomeres play an essential role in the integrity and stability of the chromosome and cell survival. ${ }^{10}$ During DNA replication in the process of cell division, telomere length (TL) can undergo a reduction of about 20 to 40 kilobase pairs (kbp) per year in peripheral blood leukocytes. Due to this mechanism, a negative correlation was observed between age and TL. ${ }^{11-13} \mathrm{TL}$ shortening is an important marker of the replicative capacity of the cell, which makes it a suitable marker of cellular aging. ${ }^{14}$ In a pathological context, inflammation and oxidative stress present a negative association with TL. ${ }^{15,16}$ Hyperglycemia is one of the most important factors that determine the production of oxidative stress and its consequent reactive oxygen species (ROS), all of which produces chronic complications in diabetes, including the shortening of TL. ${ }^{17,18}$

Although several studies have reported a negative correlation between TL and T1D or T2D, ${ }^{19}$ no studies have been conducted so far on the possible association between TL and MODY 
patients. Therefore, the hypothesis of the present work was that MODY patients may suffer a reduction in TL due to oxidative stress produced by chronic hyperglycemia, despite not presenting insulin resistance or inflammation. In reference to this, the promoter of the Creactive protein (CRP) gene requires hepatocyte nuclear factor 1 alpha (HNF1A) for its expression. Mutations present in the HNF1A gene (MODY3) are associated with reductions in CRP levels, an important marker of inflammation, compared to other types of diabetes and non-diabetic individuals. ${ }^{20,21}$

To test this hypothesis, we evaluated absolute TL (aTL) in genetically diagnosed MODY2 and MODY3 individuals and non-diabetics controls. We also assessed the correlation between aTL and other clinical or metabolic factors and possible differences in TL between MODY2 and MODY3 patients.

\section{2 | MATERIALS AND METHODS}

\subsection{Study population}

The size of the sample was calculated from a previous report which informed a $30 \%$ shorter mean telomere length in patients with type 1 diabetes when compared with controls. ${ }^{18}$ Assuming a mean telomere length of $13 \mathrm{Kpb}$ (highest mean value), with an effect size of 1.25 (delta) -corresponding with a 50\% shorter telomere length in MODY subjects, an alpha error of 0.05 and a power of $80 \%-$, a total sample size of 30 individuals was considered sufficient.

We analyzed 35 patients with genetic diagnosis of MODY2, 12 with MODY3 and 48 unrelated non-diabetic control individuals, matched by sex and age from the general population of the Autonomous City of Buenos Aires (BA) and its metropolitan area, Argentina. None of the controls had clinical components of MODY or a family history of MODY (self-reported). The individuals recruited had normal results in the medical examination and blood counts, and were not taking any medications. In this respect, the 
diabetic status was ruled out in accordance with the American Diabetes Association recommendation. ${ }^{6}$

Clinical characterization of MODY was done following the ADA (American Diabetes Association) recommendation. ${ }^{6}$ Genetic studies consisted in the purification of genomic DNA from peripheral blood by MagNA Pure system (Roche), PCR amplification of the coding regions of both genes was carried out as published by Kaisaki P. et al. and Stoffel M. et al, ${ }^{22,23}$ followed by Sanger sequencing. Mutations present in all participants were analyzed by bioinformatic tools to determine the possible effect of the alterations found. Among them, the service provided by mutation taster was preferred for being more accurate.

Glutamic acid decarboxylase autoantibodies (GADA), insulin autoantibodies (IAA) and antiphosphatase autoantibodies (IA2A) were measured by radioligand assays in all MODY patients, being in all cases negative.

Demographic data including age and gender of the patients were collected. The anthropometric measures (height, weight and waist circumference [CC]) and systolic and diastolic blood pressure (SysBP and DiasBP, respectively) were determined using a standardized protocol. The body mass index (BMI) was calculated as weight (kg) / height $(\mathrm{m})^{2}$. Fasting plasma glucose (FPG) and HA1c levels were performed by the usual standardized biochemical procedures.

\subsection{Measurement of leukocyte aTL}

The estimation of aTL was carried out using an optimized methodology based on real time polymerase chain reaction (qPCR), using a previously described protocol. ${ }^{24}$ Genomic DNA samples were purified from peripheral blood leukocytes and aTL was determined using SYBR green as an intercalating agent. This method allows us to determine the repetitive sequences of telomeres compared to a single copy gene using standard curves. For the PCR of the single- 
copy gene, a 75-bp oligonucleotide of the RPLP0 (ribosomal protein, large, subunit P0) gene was used as a standard and allowed the genome/reaction number $(\mathrm{S})$ to be exported from the standard curve. The standard for telomere PCR is a synthetic oligonucleotide of 84 bp in length formed by the tandem repeats of telomeric DNA and the standard curve is used to measure the kbp of telomeric sequence/reaction (T).

The calculation of the T/S ratio allows to determine the kbp of telomeric sequence per cell for each individual. The PCR reactions were performed in duplicate for all the study samples in a StepOne TM Real-Time PCR System (Applied Biosystems). DNA (20ng) was amplified in a $20 \mu \mathrm{l}$ reaction volume containing $10 \mu \mathrm{l}$ of SYBR Select Master Mix, and $250 \mathrm{nM}$ of primers were added for the RPLP0 gene or $100 \mathrm{nM}$ of primers for the telomeric sequence.

The PCR conditions consisted of a denaturation of $10 \mathrm{~min}$ at $95{ }^{\circ} \mathrm{C}$ followed by 40 cycles at $95^{\circ} \mathrm{C}$ for 15 seconds, $60{ }^{\circ} \mathrm{C}$ for 1 minute and the melting curve with 1 cycle of 15 seconds at $95^{\circ} \mathrm{C}, 1$ minute at $60^{\circ} \mathrm{C}$ and 15 seconds at $98^{\circ} \mathrm{C}$ with a temperature ramp of $0.3^{\circ} \mathrm{C} /$ second.

\subsection{Statistical analysis}

The statistical analysis was carried out through Statistical Package for Social Sciences software (SPSS version 20.0) with a level of significance of 0.05 . Normal distribution of continuous variables was examined by Kolmogorov-Smirnov normality test.

The relationship between aTL and clinical-biochemical and anthropometric characteristics in whole samples were assessed with partial correlation analysis using age and gender with covariates.

Comparison of clinical-biochemical and anthropometric characteristics between groups is evaluated by univariate analysis of covariance adjusting for age and gender (ANCOVA), followed by Bonferroni post hoc test for multiple comparisons. 
Among MODY patients the relationship between clinical-biochemical and anthropometric characteristics is evaluated by linear regression model or partial correlation analysis controlling for the effect of age, gender or type of MODY.

\section{3 | RESULTS}

The 95 participants of this study presented a mean age of $23.61 \pm 13.11$ years. Table 1 shows the mean clinical-biochemical and anthropometric characteristics of control, MODY2 and MODY3 patients. The groups differed significantly in weight and, in particular, MODY2 patients showed significant differences in BMI, FPG levels and DiasBP compared to controls. MODY3 patients, instead, differed significantly in FPG levels and TG_HDL respect to controls.

The complete sample showed a negative correlation between aTL and age $(r=-0.311, p=0.002$,

Figure 1A). Among the different clinical biochemical variables analyzed in the complete sample, aTL only showed a significant negative correlation with FPG $(\mathrm{r}=-0.241, \mathrm{p}=0.026)$. This correlation remained significant even when age and gender were used as covariates $(r=-$ 0.347, $\mathrm{p}=0.001)$.

Most important, MODY patients had significantly shorter mean aTL than controls $(6.49 \pm 3.31$ Kpb vs $11.13 \pm 7.82 \mathrm{Kpb}, \mathrm{p}=0.006$, Figure 1B), a difference which remained significant even after adjustment for non-modifiable factors gender and age $(\mathrm{p}<0.001)$.

Among MODY patients, a significant negative correlation was observed between aTL and age $(\mathrm{r}=-0.447, \mathrm{p}=0.009$, covariate=gender), FPG levels $(\mathrm{r}=-0.586, \mathrm{p}=0.008$, covariates=age, gender, and type of MODY) and duration of the disease ( $r=-0.385, p=0.027$, covariates=gender and type of MODY). Furthermore, we found a significant positive correlation between disease duration and weight $(\mathrm{r}=0.504, \mathrm{p}=0.005)$ and $\mathrm{BMI}(\mathrm{r}=0.504$, 
$\mathrm{p}=0.001$ ) using gender and MODY type as covariates. No significant correlations were found with metabolic parameters.

Regarding the characteristics of each MODY type, MODY3 patients exhibited significantly higher weight $(\mathrm{p}<0.001)$, BMI $(\mathrm{p}<0.001)$, SysBP $(\mathrm{p}=0.008)$ and DiasBP $(\mathrm{p}=0.021)$ than MODY2 patients (Table 1). In turn, no significant differences were found between MODY2 and MODY3 individuals in terms of aTL (Figure 2A), whereas a negative correlation was observed between aTL and age in both MODY2 $(\mathrm{r}=-0.369 \mathrm{p}=0.029)$ and MODY3 $(\mathrm{r}=-0.754$, $\mathrm{p}=0.023$ ) individuals (Figure 2B). Worth highlighting, MODY2 patients revealed a significant negative correlation between aTL and HbA1c $(r=-0.388, p=0.030)$ with gender and age as covariates (Figure 3), while MODY3 patients showed a trend toward lower aTL at higher HbA1c levels ( $r=-0.760, p=0.07)$ with gender as a covariate.

\section{4 | DISCUSSION}

TL is an important parameter which has increasingly gained importance due to its proven relationship to a variety of diseases, among them metabolic diseases such as diabetes. Given their chemical composition, telomeres are highly vulnerable to oxidative damage and inflammation, which might accelerate telomere shortening. ${ }^{25}$ In addition, hyperglycemia induces high oxidative stress, and consequent telomere shortening. ${ }^{26}$ Therefore, telomere shortening mechanisms may vary depending on the pathogenicity of diabetes.

Previous studies have shown more significant mononuclear cell telomere shortening in T1D than in T2D patients. ${ }^{27}$ In this context, the hypothesis of the present work was that MODY patients may suffer a reduction in TL due to oxidative stress produced by chronic hyperglycemia, despite not presenting insulin resistance or inflammation. To test this hypothesis, we evaluated aTL in MODY2 and MODY3 patients compared with Control subjects. 
We observed a negative correlation between aTL and age in the whole population. This result was expected, considering that age is the main factor involved in the shortening of telomeres and allowed us to validate the method. Furthermore, we found that aTL was negatively correlated with FPG levels. In agreement, TL was recently shown to be negatively associated with glucose concentrations and HbA1c within the normal non-diabetic range, ${ }^{28}$ which shows an important contribution of hyperglycemia to telomere shortening.

We found, for the first time, that MODY patients have shorter aTL with respect to non-diabetic Controls, which may be a consequence of MODY-associated hyperglycemia. We also observed a significant negative association between aTL and age among MODY patients, and in both subtypes studied, MODY2 and MODY3.

Additionally, aTL showed a negative correlation with duration of the disease and FPG levels in MODY patients in general and also with HbA1c in MODY2 patients in particular, after adjustment for age, gender and type of MODY. On the one hand, the absence of correlation between aTL and HbA1c in MODY3 patients may explained by small cohort size. On the other hand, these results find support in studies by Rosa et al., who recently showed that relative telomere length was inversely associated with FPG and HbA1c levels in patients newly diagnosed with T2D, after adjustment for age, gender and BMI, and also with duration of the disease. ${ }^{29}$ In addition, a study by Januszewski et al. revealed shorter TL in 199 T1D patients as compared to controls and a negative correlation between TL and duration of the disease and age. ${ }^{30}$ On the whole, our findings show a significant contribution of hyperglycemia and disease duration to telomere shortening even in the absence of insulin resistance or inflammation.

As opposed to what may have been expected in newly diagnosed MODY patients that have significant differences in the glucose level, ${ }^{31}$ we found no differences in aTL between our MODY2 and MODY3 patients, whose glucose levels were controlled through treatment. It can be thus inferred that both MODY types present telomere shortening which, at least partly, 
responds to HbA1c and FPG levels, which suggests comparable mechanisms underlying the attrition of TL.

The main limitation is that the sample size was relatively small; therefore, the results of this study should be verified using a larger sample size. We cannot exclude that some clinical characteristics such as inflammation or lifestyle markers that were not available for analysis could affect aTL. Furthermore, being a cross-sectional study, the present work cannot address the rate of telomere attrition in MODY patients.

Taken together, our findings on aTL in MODY patients may provide a parameter relatively easy and inexpensive to quantify the impact of the high glucose level, in order to carry out antidiabetic treatments with strict targets.

\section{Conflicts of Interest}

We wish to confirm that there are no known conflicts of interest associated with this publication and there has been no financial support for this work that could have influenced its outcome.

\section{Ethical Declaration}

This work has been approved by the Institutional Review Committee and written informed consent was obtained from all individuals involved or from responsible family members after full explanation of the purpose and nature of all procedures used. All human investigations were conducted according to the principles expressed in the Declaration of Helsinki as revised in 1983. Informed consent was obtained from all individual participants included in the study.

\section{Contribution statement}


ALM performed the most part of the laboratory work, designed the research study, analyzed the data and wrote the paper. SIT provided the control individuals samples and the clinical data for the study. MCG performed part of the laboratory work related to the control individuals. ADD provided the patients samples and the clinical data for the study. GEC assisted in the design of the research study and analyzed the data. GDF designed the research study and supervised the writing of the paper. APL provided the funds, designed the research study and supervised the writing of the paper. All authors declare to have been revising the paper critically and have been approved the final version. 


\section{REFERENCES}

1. Kim SH. Maturity-Onset Diabetes of the Young: What Do Clinicians Need to Know? Diabetes Metab J. 2015;39(6):468-77.

2. Timsit J, Bellanné-Chantelot C, Dubois-Laforgue D, Velho G. Diagnosis and management of maturity-onset diabetes of the young. Treat Endocrinol. 2005;4(1):9-18

3. Thanabalansingham G, Pal A, Selwood MP, et al. Systematic assessment of etiology in adults with a clinical diagnosis of young-onset type 2 diabetes is a successful strategy for identifying maturity-onset diabetes of the young. Diabetes Care. 2012;35:1206-1212

4. Shields BM, Hicks S, Shepherd MH, Colclough K, Hattersley AT, Ellard S. Maturity-onset diabetes of the young (MODY): how many cases are we missing? Diabetologia. 2010;53(12):2504-8.

5. World Health Organization. Definition and Diagnosis of Diabetes Mellitus and Intermediate Hyperglycaemia. Geneva, Switzerland: World Health Organization (2006).

6. Classification and Diagnosis of Diabetes: Standards of Medical Care in Diabetes 2020. American Diabetes Association. Diabetes Care 2020 Jan; 43 (Supplement 1): S14-S31.

7. Velho G, Froguel P. Genetic, metabolic and clinical characteristics of maturity onset diabetes of the young. Eur J Endocrinol. 1998;138(3):233-9

8. Stoffel M, Froguel P, Takeda J, et al. Human glucokinase gene: isolation, characterization, and identification of two missense mutations linked to early-onset non-insulin-dependent (type 2) diabetes mellitus. Proc Natl Acad Sci USA. 1992;89(16):7698-702.

9. Fajans SS, Bell GI, Polonsky KS. Molecular mechanisms and clinical pathophysiology of maturity-onset diabetes of the young. N Engl J Med. 2001;345(13):971-80.

10. Blackburn EH. Telomere states and cell fates. Nature. 2000;408(6808):53-6 
11. Hastie ND, Dempster M, Dunlop MG, Thompson AM, Green DK, Allshire RC. Telomere reduction in human colorectal carcinoma and with ageing. Nature. 1990;346(6287):866-8.

12. Iwama H, Ohyashiki K, Ohyashiki JH, et al. Telomeric length and telomerase activity vary with age in peripheral blood cells obtained from normal individuals. Hum Genet. 1998;102(4):397-402.

13. Satoh H, Hiyama K, Takeda M, et al. Telomere shortening in peripheral blood cells was related with aging but not with white blood cell count. Jpn J Hum Genet. 1996;41(4):413-7.

14. Mikhelsol VM, Gamaley IA. Telomere Shortening is a Sole Mechanism of Aging in Mammals. Curr Aging Sci. 2012;5(3):203-8

15. Barnes RP, Fouquerel E, Opresko PL. The impact of oxidative DNA damage and stress on telomere homeostasis. Mech Ageing Dev. 2019;177:37-45.

16. Jurk D, Wilson C, Passos JF, et al.. Chronic inflammation induces telomere dysfunction and accelerates ageing in mice. Nat Commun. 2014;24;2:4172

17. Fiorentino TV, Prioletta A, Zuo P, Folli F. Hyperglycemia-induced oxidative stress and its role in diabetes mellitus related cardiovascular diseases. Curr Pharm Des. 2013;19(32):5695-703.

18. Ma D, Zhu W, Hu S, Yu X, Yang Y. Association between oxidative stress andtelomere length in Type 1 and Type 2 diabetic patients. J Endocrinol Invest. 2013;36(11):1032-7.

19. Wang J, Dong X, Cao L, et al. Association between telomere length and diabetes mellitus: A meta-analysis. J Int Med Res. 2016;44(6):1156-1173.

20. Owen KR, Thanabalasingham G, James TJ, Karpe F, Farmer AJ, McCarthy MI, Gloyn AL. Assessment of high-sensitivity C-reactive protein levels as diagnostic discriminator of maturity-onset diabetes of the young due to HNF1A mutations. Diabetes Care. 2010 Sep;33(9):1919-24 
21. Thanabalasingham, G., et al., A large multicentre European study validates highsensitivity $\mathrm{C}$ reactive protein (hsCRP) as a clinical biomarker for the diagnosis of diabetes subtypes. Diabetologia, 2011. 54(11): p. 2801-10.

22. Kaisaki PJ, Menzel S, Lindner T, et al. Mutations in the hepatocyte nuclear factor-1alpha gene in MODY and early-onset NIDDM: evidence for a mutational hotspot in exon 4 . Diabetes. 1997;46(3):528-35.

23. Stoffel M, Patel P, Lo YM, et al. Missense glucokinase mutation in maturity-onset diabetes of the young and mutation screening in late-onset diabetes. Nat Genet. 1992;2(2):153-6

24. O’Callaghan NJ, Fenech M. A quantitative PCR method for measuring absolute telomere length. Biol Proced Online. 2011;13:3.

25. Koliada AK, Krasnenkov DS, Vaiserman AM. Telomeric aging: mitotic clock or stress indicator? Front Genet. 2015;16;6:82.

26. Salpea KD, Talmud PJ, Cooper JA, et al. Association of telomere length with type 2 diabetes, oxidative stress and UCP2 gene variation. Atherosclerosis. 2010;209(1):42-50

27. Jeanclos E, Krolewski A, Skurnick J, et al. Shortened telomere length in white blood cells of patients with IDDM. Diabetes. 1998;47(3):482-6.

28. Grunnet LG, Pilgaard K, Alibegovic A, et al. Leukocyte telomere length is associated with elevated plasma glucose and HbA1c in young healthy men independent of birth weight. Sci Rep. 2019;9(1):7639.

29. Rosa ECCC, Dos Santos RRC, Fernandes LFA, Neves FAR, Coelho MS, Amato AA. Leukocyte telomere length correlates with glucose control in adults with recently diagnosed type 2 diabetes. Diabetes Res Clin Pract. 2018;135:30-36. 
30. Januszewski AS, Sutanto SS, McLennan S, et al. Shorter telomeres in adults with Type 1 diabetes correlate with diabetes duration, but only weakly with vascular function and risk factors. Diabetes Res Clin Pract. 2016;117:4-11.

31. Urakami T. Maturity-onset diabetes of the young (MODY): current perspectives on diagnosis and treatment. Diabetes Metab Syndr Obes. 2019;12:1047-1056 
Table1. Clinical-biochemical and anthropometric characteristics of Control and patients with MODY2 and MODY3.

\begin{tabular}{|c|c|c|c|c|c|c|c|}
\hline & Control & MODY2 & MODY3 & $\mathbf{P}$ & $\begin{array}{c}\mathbf{p} \\
\text { Control vs. } \\
\text { MODY2 }\end{array}$ & $\begin{array}{c}\mathbf{p} \\
\text { Control vs. } \\
\text { MODY3 }\end{array}$ & $\begin{array}{c}\text { p } \\
\text { MODY2 vs. } \\
\text { MODY3 }\end{array}$ \\
\hline n & 48 & 35 & 12 & & & & \\
\hline Age (years) & $24 \pm 9.66$ & $20.11 \pm 14.43$ & $30.25 \pm 18.42$ & 0.035 & 0.028 & 0.049 & 0.161 \\
\hline aTL (Kpb) & $11.13 \pm 7.82$ & $6.64 \pm 3.50$ & $6.05 \pm 2.78$ & $<0.001$ & $<0.001$ & 0.002 & 0.645 \\
\hline Weight (kg) & $65.43 \pm 12.00$ & $45.38 \pm 17.52$ & $67.19 \pm 14.49$ & $<0.001$ & $<0.001$ & 0.029 & $<0.001$ \\
\hline WC (cm) & $77.38 \pm 10.42$ & $68 \pm 23.42$ & $86.33 \pm 22.05$ & 0.180 & 0.343 & 0.156 & 0.634 \\
\hline BMI (kg/m2) & $23.45 \pm 3.93$ & $19.82 \pm 3.34$ & $24.02 \pm 3.56$ & $<0.001$ & $<0.001$ & 0.687 & $<0.001$ \\
\hline FPG (mg/dl) & $85.3617 \pm 8.98$ & $117.40 \pm 11.59$ & $129.37 \pm 14.12$ & $<0.001$ & $<0.001$ & $<0.001$ & 0.095 \\
\hline TG(mg/dl) & $77.15 \pm 34.17$ & $87.31 \pm 62.26$ & $125.62 \pm 93.22$ & 0.046 & 0.446 & 0.050 & 0.181 \\
\hline TC (mg/dl) & $157.58 \pm 29.03$ & $170.35 \pm 52.24$ & $178.5 \pm 27.52$ & 0.222 & 0.144 & 0.179 & 0.318 \\
\hline LDL-C (mg/dl) & $87.04 \pm 22.81$ & $98.37 \pm 39.34$ & $101.37 \pm 22.63$ & 0.150 & 0.125 & 0.131 & 0.423 \\
\hline HDL-C (mg/dl) & $56.50 \pm 14.53$ & $53.32 \pm 12.62$ & $60.25 \pm 12.57$ & 0.383 & 0.432 & 0.309 & 0.451 \\
\hline TG/HDL & $1.44 \pm 0.78$ & $1.75 \pm 1.27$ & $2.25 \pm 1.85$ & 0.031 & 0.186 & 0.004 & 0.270 \\
\hline HbA1c (\%) & & $6.32 \pm 0.42$ & $6.92 \pm 1.56$ & - & - & - & 0.139 \\
\hline SysBP (mmHg) & $113.18 \pm 8.53$ & $107.42 \pm 8.39$ & $118.50 \pm 11.36$ & 0.049 & 0.105 & 0.567 & 0.008 \\
\hline DiasBP (mmHg) & $70.68 \pm 6.78$ & $60.33 \pm 6.21$ & $77 \pm 12.49$ & 0.001 & 0.001 & 0.350 & 0.021 \\
\hline
\end{tabular}

Values are expressed as mean \pm standard deviation (SD). P and p values were obtained from univariate analysis adjusted by age and gender (ANCOVA). Data with significant differences within groups $(\mathrm{p}<0.05)$ are in bold. aTL: absolute telomere length, WC: waist circumference, BMI: body mass index, FPG: fasting plasma glucose, TG: triglycerides, TC: total cholesterol, LDL-C: low density 
cholesterol, HDL-C: high density cholesterol, HbA1c: glycated hemoglobin A1c, SysBP: systolic blood pressure, DiasBP: diastolic blood pressure. 

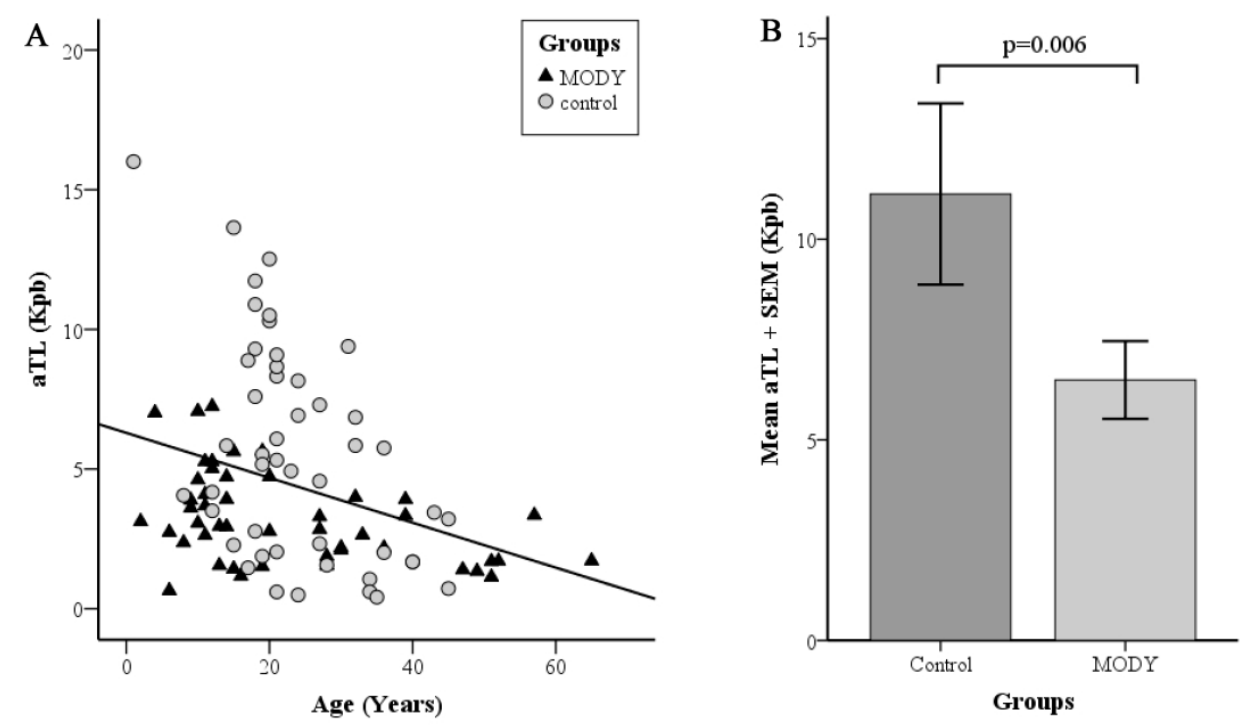

Figure 1: Relationship of absolute telomere length with age and MODY. (A) Distribution of Absolute Telomere length according to age in Control and MODY groups. The linear regression between age and the aTL in the whole samples ( $R 2=0.120$, Pearson correlation coefficients $r=-0.320, p=0.002$ ) is shown. (B) Bar graphs illustrating mean \pm standard error of mean (SEM) of aTL in controland type MODY patients. $p<0.05$ was considered as significant. aTL: Absolute telomere length

$$
162 \times 91 \mathrm{~mm}(300 \times 300 \mathrm{DPI})
$$



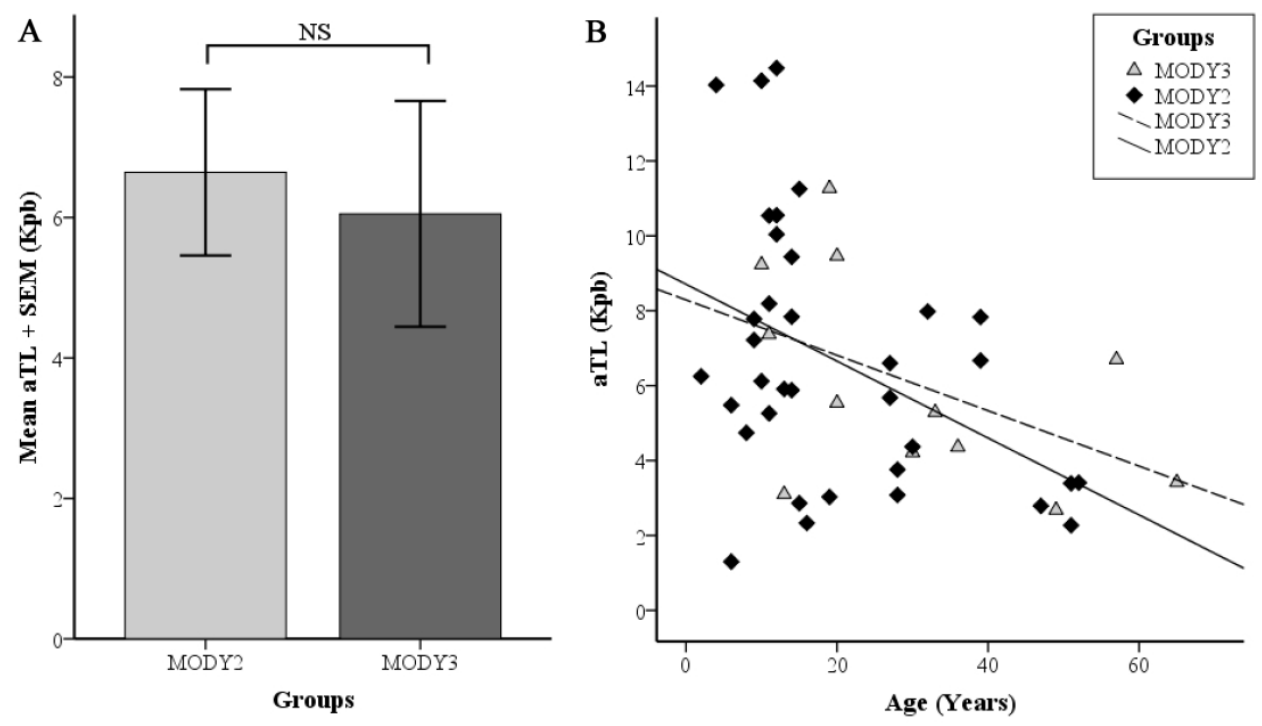

Figure 2: Relationship of absolute telomere length with MODY type and age. (A) Bar graphs illustrating mean \pm standard error of mean (SEM) of aTL in MODY $2(n=35)$ and MODY $3(n=12)$. aTL: Absolute telomere length. NS: no significate. (B) Distribution of aTL according to age. Linear regression model between aTL and age in MODY2 $(R 2=0.136, p=0.035)$ and MODY3 $(R 2=0.569, p=0.023) . p<0.05$ was considered significant.

$162 \times 91 \mathrm{~mm}(300 \times 300 \mathrm{DPI})$ 


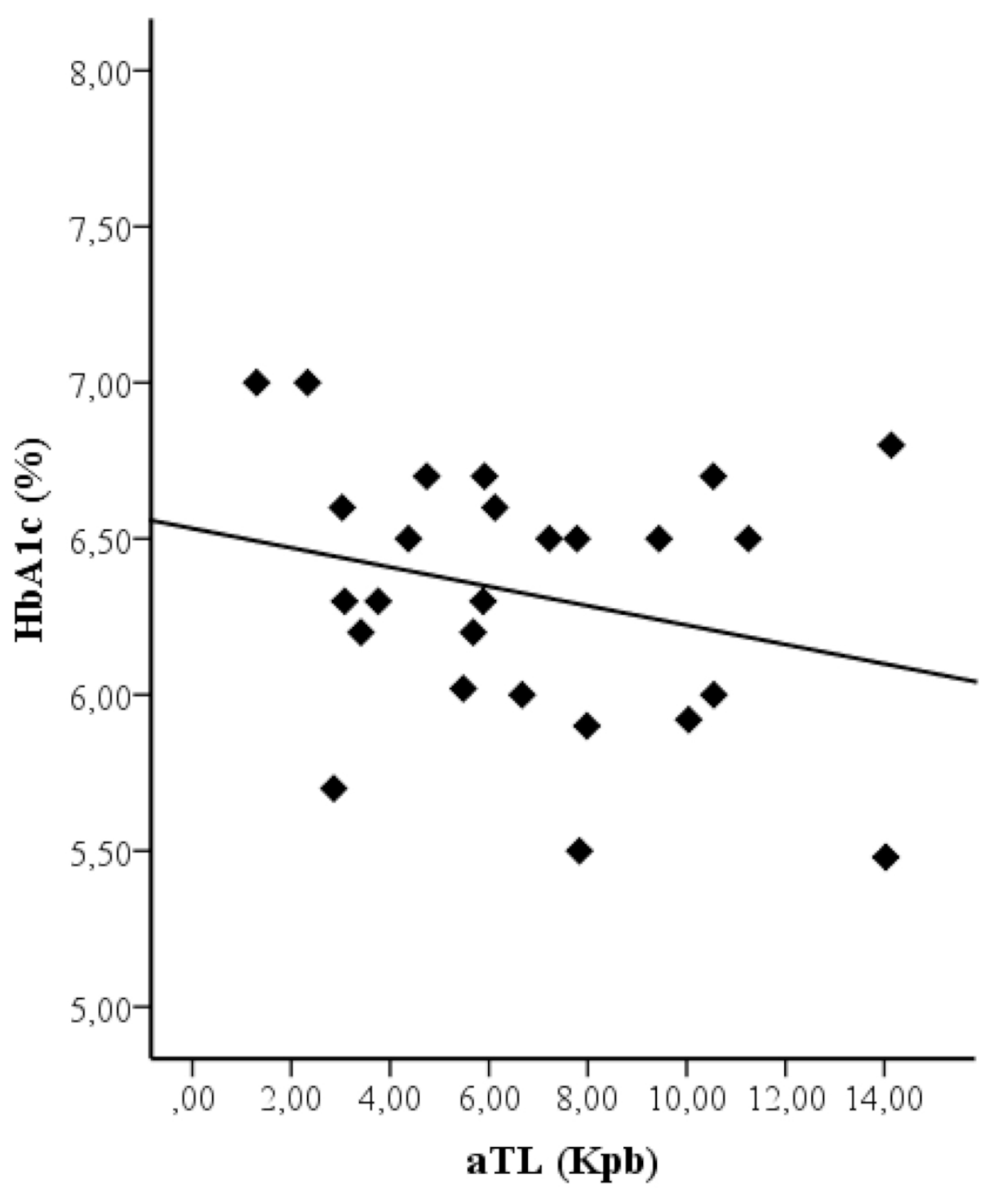

Figure 3: Relationship of absolute telomere length with HbA1c in MODY2 patients. Linear correlation between $\mathrm{aTL}$ and HbA1c $(\%)$ in MODY2 patients $(r=-0.388, p=0.030$, covariates $=$ age and gender).

$$
162 \times 184 \mathrm{~mm}(300 \times 300 \mathrm{DPI})
$$

